\title{
Molecular Dynamics Study on the Effect of Temperature on the Tensile Properties of Single-Walled Carbon Nanotubes with a Ni-Coating
}

\author{
Fulong Zhu, ${ }^{1}$ Hengyou Liao, ${ }^{2}$ Kai Tang, ${ }^{1}$ Youkai Chen, ${ }^{1}$ and Sheng Liu ${ }^{1}$ \\ ${ }^{1}$ Institute of Microsystems, School of Mechanical Science and Engineering, Huazhong University of Science and Technology, \\ 1037 Luoyu Road, Wuhan, Hubei 430074, China \\ ${ }^{2}$ Dongfang Electric Machinery Co., Ltd, Deyang, Sichuan 618000, China \\ Correspondence should be addressed to Fulong Zhu; zhufulong@hust.edu.cn
}

Received 16 September 2014; Accepted 18 January 2015

Academic Editor: Cheng Yan

Copyright (C) 2015 Fulong Zhu et al. This is an open access article distributed under the Creative Commons Attribution License, which permits unrestricted use, distribution, and reproduction in any medium, provided the original work is properly cited.

\begin{abstract}
The effect of temperature on the tensile behavior of the armchair $(6,6)$ single-walled carbon nanotubes with a Ni-coating (SWCNT$\mathrm{Ni}$ ) was investigated using molecular dynamics (MD) methods. The mechanical properties of SWCNT-Ni and SWCNT were calculated and analyzed at different temperatures in the range from $220 \mathrm{~K}$ to $1200 \mathrm{~K}$. From the MD results, temperature was determined to be the crucial factor affecting the mechanical properties of SWCNT-Ni and SWCNT. After coating nickel atoms onto the surface of a SWCNT, the Young's modulus, tensile strength, and tensile failure strain of SWCNT were greatly reduced with temperature rising, indicating that the nickel atoms on the surface of SWCNT degrade its mechanical properties. However, at high temperature, the Young's modulus of both the SWCNT and the SWCNT-Ni exhibited significantly greater temperature sensitivity than at low temperatures, as the mechanical properties of SWCNT-Ni were primarily dominated by temperature and $\mathrm{C}-\mathrm{Ni}$ interactions. During these stretching processes at different temperatures, the nickel atoms on the surface of SWCNT-Ni could obtain the amount of energy sufficient to break the $\mathrm{C}-\mathrm{C}$ bonds as the temperature increases.
\end{abstract}

\section{Introduction}

Since discovery in 1991 [1] and due to the excellent properties such as high thermal and electrical conductivity, ultra mechanical strength, low density, and high aspect ratio, carbon nanotubes (CNTs) have gained increasing attention from both academia and industry. The thermal conductivity of multiwalled carbon nanotubes (MWCNT) can reach up to $3000 \mathrm{~W} / \mathrm{mK}$ [2], and the thermal conductivity of singlewalled nanotubes (SWCNT) can be even higher [3]. Some types of CNTs are considered to be ideal candidates as reinforcing materials to enhance the properties of composites and electrical and thermal devices $[4,5]$.

Because of the remarkable mechanical properties of SWCNT, it is believed that the mechanical properties of a metal matrix can be greatly improved by mixing the metal with SWCNT coated by a metal film, such as nickel or copper. For some types of metals, during mixing, the metal could agglomerate into discrete deposits on SWCNT bundles [6,7], which could decrease the adhesive force between SWCNT and the metal matrix, thus preventing the improvement of the mechanical properties of the metal by mixing the metal with SWCNTs. To increase the adhesive force between SWCNT and some metal atoms, the surface of each SWCNT must be treated by coating some metal films, such as nickel or copper. After coating certain metal films onto the surfaces of SWCNTs, the SWCNTs with a coating of metal film are suitable to mix with the metal matrix, thereby improving the efficiency of load transfer from the metal matrix to the carbon nanotubes. Coating nickel or copper is one conventional method of decorating the surface of SWCNT. Nickel atoms can be coated onto suspended single-walled carbon nanotubes using electron-beam evaporation, as the coating of nickel atoms on the suspended carbon tubes has been demonstrated to be continuous and quasi-continuous [6]. Some metals were also deposited onto a vertically aligned 
SWCNT (VA-SWCNT) film by evaporative deposition [7]; and the continuous spreading of nickel atoms onto carbon nanotubes was investigated using molecular dynamics methods [8]. Some experiments also indicated that the mechanical and tribological properties of the copper matrix composites could be significantly enhanced by the incorporation of SWCNT reinforcements after coating SWCNT with nickel metal [9]. Meanwhile, the decorated CNTs could also be functionalized to satisfy interconnecting processing or for the application of nanoscale sensors [10].

To determine the effect of temperature on the mechanical properties of armchair $(6,6)$ single-walled carbon nanotubes with a Ni-coating (SWCNT-Ni), a perfect SWCNT decorated with a coating of nickel atoms on its surfaces is considered in this work. Although the influence of nickel atoms on SWCNT was investigated at $0 \mathrm{~K}$ and $300 \mathrm{~K}$ [11], a wide temperature range will be defined in this paper by considering temperatures of $220 \mathrm{~K}, 300 \mathrm{~K}, 500 \mathrm{~K}, 700 \mathrm{~K}, 1000 \mathrm{~K}$, and $1200 \mathrm{~K}$. After modeling and analyzing the stretching process of SWCNT and SWCNT-Ni at different temperatures of $220 \mathrm{~K}, 300 \mathrm{~K}$, $500 \mathrm{~K}, 700 \mathrm{~K}, 1000 \mathrm{~K}$, and $1200 \mathrm{~K}$ using molecular dynamics methods, the mechanical behaviors of the SWCNT and the SWCNT-Ni can then be determined. In the following molecular dynamics simulation for stretching SWCNT and SWCNT-Ni, a more complicated second-generation Brenner reactive empirical bond-order potential will be introduced for analyzing the behaviors of SWCNT-Ni and SWCNT. In the following section, the stress-strain curves of SWCNT and SWCNT-Ni are determined with the data from these molecular dynamics computational results, their fracture surfaces are also determined, and the influence of temperature on the coating of nickel onto the surface of SWCNT is revealed.

\section{Computational Methods and Models}

To investigate the effect of nickel atoms and temperature on the mechanical behavior of SWCNT-Ni, the surface of an armchair $(6,6)$ SWCNT was coated with nickel using metal cluster deposition [8]. After coating nickel atoms onto the surface of SWCNT, nickel clusters were demonstrated to spread and form a continuous smooth surface of a VA-SWCNT, in which the equilibrium position for nickel atoms was the center of the hexagons of the carbon atoms of SWCNT [12]. In this work, molecular dynamics simulations of the stretching process at different temperatures for SWCNT-Ni and SWCNT were performed, based on the large-scale atomic/molecular massively parallel simulator (LAMMPS) code [13]. In the molecular dynamics (MD) modeling of SWCNT and SWCNT-Ni, to simplify the cluster deposition process, nickel atoms were simply placed in the vicinity of the center of a carbon hexagon, with every computing step of the molecular dynamics simulation following a period of energy minimization; MD simulations for the stretching process of SWCNT-Ni and SWCNT were conducted at different temperatures of $220 \mathrm{~K}, 300 \mathrm{~K}, 500 \mathrm{~K}$, $700 \mathrm{~K}, 1000 \mathrm{~K}$, and $1200 \mathrm{~K}$, and Figures 1 and 2 show the stretching models. In the stretching diagrams of Figures 1 and 2 , the molecular model of the armchair $(6,6)$ SWCNT consisted of 576 carbon atoms, and the molecular model of the armchair $(6,6)$ SWCNT-Ni had 576 carbon atoms and 264 nickel atoms. In the two models shown in Figures 1 and 2, the first two rings of atoms located at each model's right end were fixed, the last two rings of atoms located at the left end were also restricted in their $x$ - and $y$ directions, and the stretching process occurred along the $z$ direction. In the MD simulations for SWCNT and SWCNT$\mathrm{Ni}$, the entire stretching process occurred in an isothermal environment; the $\mathrm{C}-\mathrm{C}$ interactions were modeled using the second-generation Brenner reactive empirical bond-order potential [14], which has been successfully used to investigate various carbon and hydrocarbon systems; the $\mathrm{C}-\mathrm{Ni}$ and $\mathrm{Ni}$ $\mathrm{Ni}$ interaction parameters were also determined using the Brenner-type potential, and its potential parameters were used from these results [14]. This type of potential could be described by the following formulae:

$$
\begin{aligned}
& E_{i j}^{\mathrm{C}-\mathrm{Ni}}= \frac{D_{e}}{S-1} \exp \left\{-\beta \sqrt{2 S}\left(r_{i j}-R_{e}\right)\right\} \\
&-B^{*} \frac{D_{e} S}{S-1} \exp \left\{-\beta \sqrt{2 / S}\left(r_{i j}-R_{e}\right)\right\}, \\
& B^{*}=\left\{1+b\left(N^{\mathrm{C}}-1\right)\right\}^{\delta}, \\
& E_{i j}^{\mathrm{Ni}-\mathrm{Ni}}= \frac{D_{e}}{S-1} \exp \left\{-\beta \sqrt{2 S}\left(r_{i j}-R_{e}\right)\right\}, \\
&-\frac{D_{e} S}{S-1} \exp \left\{-\beta \sqrt{2 / S}\left(r_{i j}-R_{e}\right)\right\}, \\
& D_{e}= D_{e 1}+D_{e 2} \exp \left\{-\mathrm{C}_{D}\left(N^{\mathrm{M}}-1\right)\right\}, \\
& R_{e}= R_{e 1}-R_{e 2} \exp \left\{-\mathrm{C}_{R}\left(N^{\mathrm{M}}-1\right)\right\}, \\
& N_{i}^{\mathrm{C}}=1+\sum_{\text {carbon }} f\left(r_{i k}\right), \\
& N_{i}^{\mathrm{M}}=1+\sum_{\text {metal }} f\left(r_{i k}\right) .
\end{aligned}
$$

Here, $r_{i j}$ was the distance between the two atoms $i$ and j. $D_{e}$ and $R_{e}$ were the binding energy and equilibrium bond length, respectively. $S, \beta, b$, and $\delta$ determined the shape of the potential functions. For the $\mathrm{C}-\mathrm{Ni}$ interactions, the manybody effects were defined by using an additional term $B^{*}$, which was expressed as a function of the carbon coordination number of a metal atom $N^{\mathrm{C}}$. For the Ni-Ni interactions, the binding energy $D_{e}$ and the equilibrium bond length $R_{e}$ were expressed as direct functions of the metal coordination number $N^{\mathrm{M}}$. Consider

$$
f\left(r_{i j}\right)= \begin{cases}1 & r<R_{1}, \\ \frac{1}{2}\left[1+\cos \frac{\pi\left(r_{i j}-R_{1}\right)}{R_{2}-R_{1}}\right] & R_{1} \leq r \leq R_{2}, \\ 0 & r>R_{2} .\end{cases}
$$

The cutoff function equation in the second-generation Brenner reactive empirical bond-order potential introduced a dramatic increase in the interatomic force at $r=R_{1}$ (similar to a camelback on the force curve) [15], which could rise 


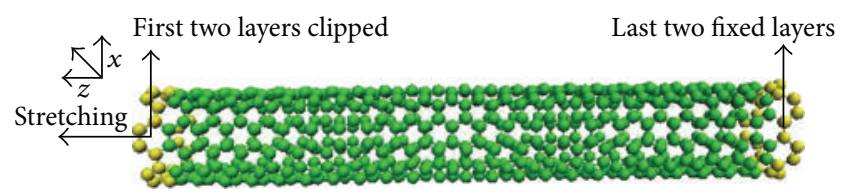

FIGURE 1: Stretching model diagram of a SWCNT.

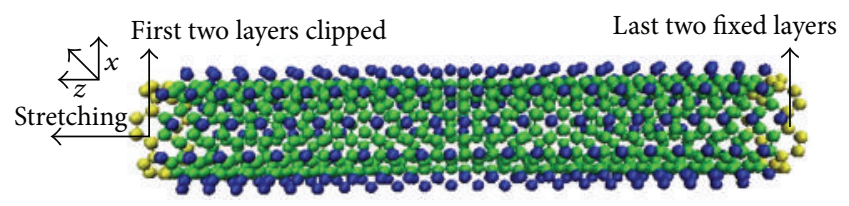

FIGURE 2: Stretching model diagram of a SWCNT-Ni.

sharply with a peak near the bond breaking length. Therefore, $R_{1}=R_{2}=2.0 \AA$ [16] was used to avoid overestimating the force in the molecular dynamics simulation of this paper.

In this work, the structure of SWCNT-Ni was two concentric cylinders, and the cross-sectional of SWCNT-Ni was two concentric circles; the cross-sectional area of SWCNT$\mathrm{Ni}$ was defined by the area between SWCNT and nickel layer, and the thickness of SWCNT-Ni was the distance between SWCNT and nickel layer. The distance between SWCNT and nickel layer was $1.5 \AA$ and the diameter of $(6,6)$ SWCNT was $8.14 \AA$. So the cross-sectional area of SWCNT-Ni was defined as $93.74 \AA \times \AA$. In the atomistic simulation, the stress of $\sigma_{m n}$ on the $m$ and $n$ directions is given by the following formula [17]:

$$
\sigma_{m n}=\frac{1}{N_{\mathrm{S}}} \sum_{i}\left[\frac{m_{i} v_{i}^{m} v_{i}^{n}}{V_{i}}-\frac{1}{2 V_{i}} \sum_{j} \frac{\partial U\left(r_{i j}\right)}{\partial r_{i j}} \frac{r_{i j}^{m} r_{i j}^{n}}{r_{i j}}\right],
$$

where $N_{\mathrm{S}}$ is the number of particles contained in the region $S$, and $S$ is defined as the region of atomic interaction, $v_{i}$ is the velocity of the particle $i$, and $r_{i j}^{m}$ and $r_{i j}^{n}$ are two components of the vector from the atoms $i$ to $j . V_{i}$ is the volume assigned to atom $i$.

For each atomic model from this work, the constant pressure and constant temperature (NPT) integration was performed using a Nose/Hoover temperature thermostat and a Nose/Hoover pressure barostat, and the bulk pressure was defined as zero. Subsequently, under the conditions of constant volume and constant temperature (NVT), these models were equilibrated to the desired temperatures using a Nose/Hoover temperature thermostat. The velocity Verlet method was used to integrate the classical equations of motion with a time step of $0.5 \mathrm{fs}$.

During the entire stretching process shown in Figures 1 and 2 , the 47 th and 48th rings of carbon atoms at the right end of both models were fixed, the first and second rings of carbon atoms at the left end were also restricted in their $x$ - and $y$ directions, and the models of SWCNT and SWCNT-Ni were gradually stretched along the $z$-direction; the tensile load was applied along the $z$-direction. After every step of stretching, it was necessary that the simulation system provide sufficient time for the model to relax. In the modeling of the stretching
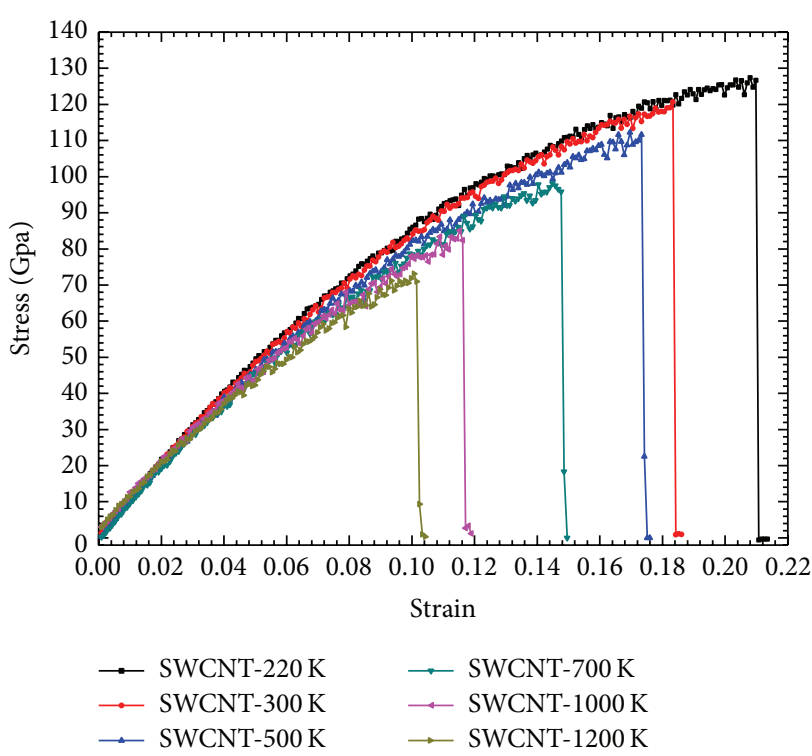

FIGURE 3: Stress-strain curves of SWCNT at different temperatures.

process, the relaxation time was defined to be $10 \mathrm{ps}$. The external applied force was obtained by summing the internal forces acting on the atoms of the stretching section. All the data used to evaluate the mechanical properties of SWCNT and SWCNT-Ni was extracted from the back 1000 steps after the relaxation time of every instance of stretching. Considering unintentional sudden variations, the average of 10 sets of data at an interval of 100 steps during the above 1000 steps was used to determine the mechanical properties of SWCNT and SWCNT-Ni.

\section{Results and Discussions}

After the MD computations of the stretching process for SWCNT and SWCNT-Ni at the different temperatures of $220 \mathrm{~K}, 300 \mathrm{~K}, 500 \mathrm{~K}, 700 \mathrm{~K}, 1000 \mathrm{~K}$, and $1200 \mathrm{~K}$, the internal forces acting on the atoms and the stretching distance were obtained, which could be transformed into the corresponding stress and strain values. Due to the difficulty of defining the thickness of SWCNT-Ni, the definition of stress in the stretching process was from the atomistic stress of the MD modeling [17]. Figures 3 and 4 show all the simulated stressstrain curves over the prescribed temperature range for SWCNT and SWCNT-Ni, which clearly indicates that temperature was the crucial factor that determines the diversity of the stress-strain curves. From the curves in Figure 4, the stress-strain curves of SWCNT-Ni over the temperature range underwent some phase of linear elastic deformation, nonlinear elastic deformation, yield deformation, strengthening, and rupture, for which the length of any phase exhibited the temperature effect. Compared with SWCNT-Ni's stressstrain curves in Figure 4, SWCNT's stress-strain curves in Figure 3 were different. During the stretching process over all the prescribed temperatures, the stress-strain curves of the SWCNT did not exhibit any yielding phase. Unlike SWCNT's stress-strain curves, the stress-strain curve of the 


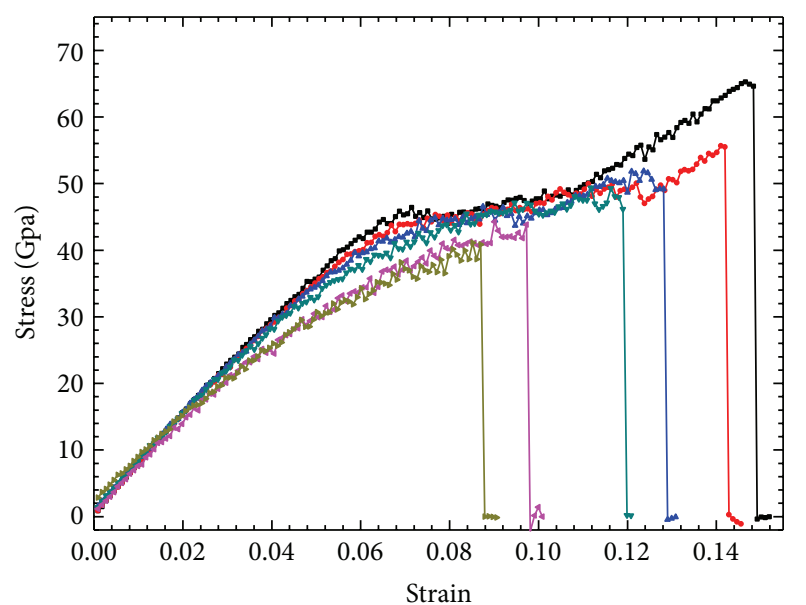

$\rightarrow$ SWCNT-Ni-220K $\rightarrow$ SWCNT-Ni-700 K
$\rightarrow$ SWCNT-Ni-300 K $\rightarrow$ SWCNT-Ni-1000 K
$\rightarrow$ SWCNT-Ni-500 K $\rightarrow$ SWCNT-Ni-1200 K

FIGURE 4: Stress-strain curves of SWCNT-Ni at different temperatures.

SWCNT-Ni at $220 \mathrm{~K}$ clearly exhibited a yielding section after elastic deformation in Figure 4, which was also found in the other curves of SWCNT-Ni. In Figure 4, SWCNT-Ni's stressstrain curves were different from those of SWCNT, which revealed that the nickel atoms coated onto the surface of SWCNT play an important role in affecting the mechanical behavior of SWCNT-Ni. In SWCNT-Ni's stretching process at different temperatures, nickel atoms' rearrangement can occur, which would change some mechanical properties of SWCNT-Ni. The strain-stress curve of the armchair (5, 5) carbon nanotubes with nickel-coating exhibited a flat phase at the beginning of the stretching at $300 \mathrm{~K}$ [11]; this phenomenon did not occur in the stress-strain curves shown in Figure 4, which might be due to the use of a different initial equilibrium method and the change of the cutoff function. In this work, NPT integration was performed using a Nose/Hoover temperature thermostat and Nose/Hoover pressure barostat, the bulk pressure was defined as zero, and the internal stress could be released fully.

According to the force and deformation data from the $\mathrm{MD}$ computations for the stretching at different temperatures, the various curves, such as the failure force and temperature, the failure stress and temperature, the failure strain and temperature, and Young's modulus and temperature, could be analyzed, thus enabling the mechanical properties of SWCNT and SWCNT-Ni to be determined at the different temperatures. Figures 5, 6, 7, and 8 show the failure force, failure stress, failure strain, and Young's modulus, respectively, at temperatures of $220 \mathrm{~K}, 300 \mathrm{~K}, 500 \mathrm{~K}, 700 \mathrm{~K}, 1000 \mathrm{~K}$, and $1200 \mathrm{~K}$. In the failure force and temperature curves in Figure 5, the failure force of SWCNT and SWCNT-Ni decreases as the temperature increases. In the same way, this tendency of decreasing as the temperature increases was observed in the curves of failure stress, failure strain, and Young's modulus in Figures 6, 7, and 8, respectively.

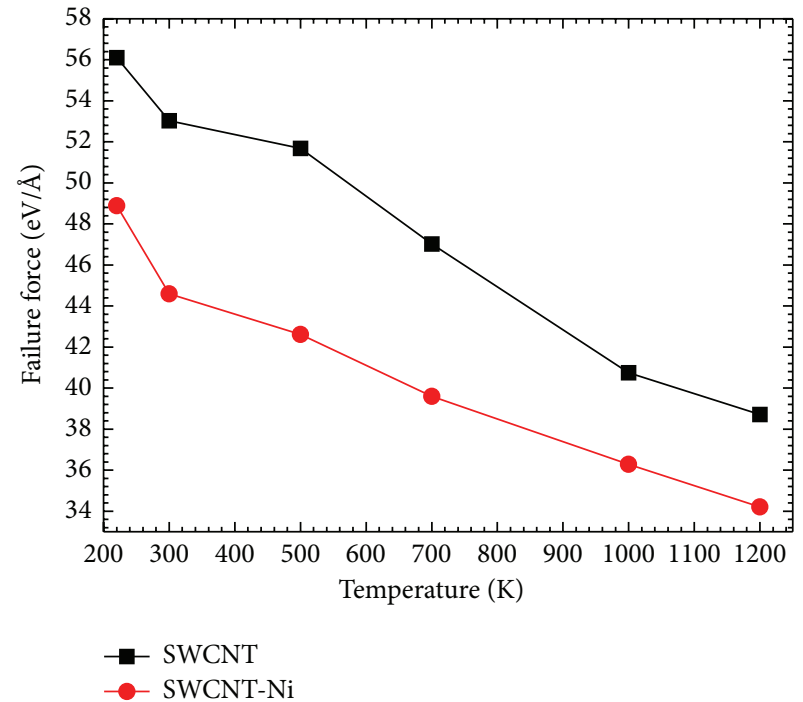

FIGURE 5: Failure force versus temperature curves of SWCNT and SWCNT-Ni.

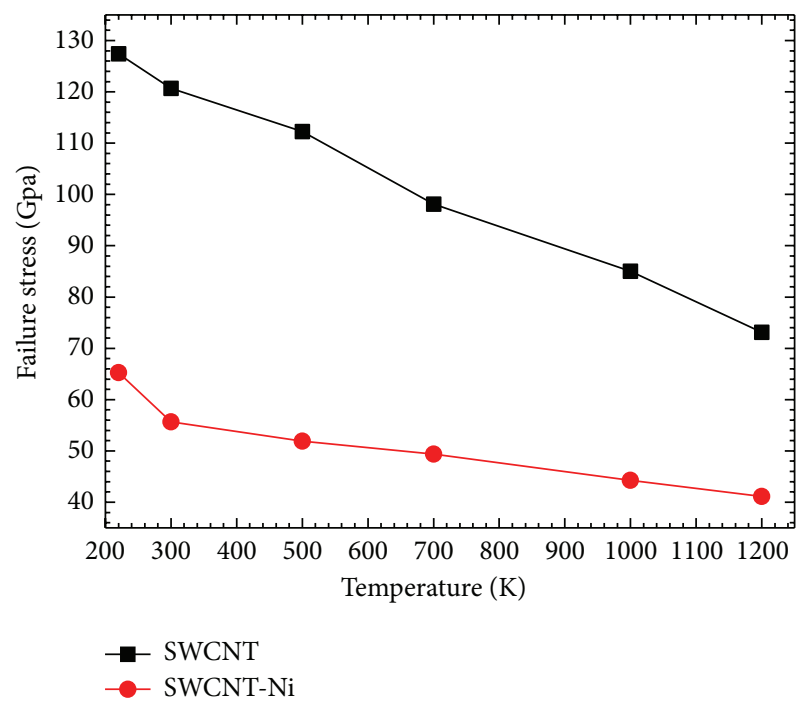

FIGURE 6: Failure stress versus temperature curves of SWCNT and SWCNT-Ni.

As shown in Figure 6, the failure stress of SWCNT decreases more rapidly than that of SWCNT-Ni as the temperature increases. In the MD computation in this work, the attenuation distance $R_{1}$ of the cutoff function increased and the failure stress of SWCNT was still higher than the experimental values that range from $11 \mathrm{GPa}$ to $63 \mathrm{GPa}$ at room temperature [18], which resulted from the perfect SWCNT used in the molecular dynamics modeling in this work. At the same time, the failure stress at different temperatures in Figure 6 was still larger than that of the modeling for a $(5,5)$ pristine SWCNT using a modified Tersoff-Brenner potential [19], which could be caused by differences in the SWCNT modeled. In Figure 6, the failure stress of SWCNT$\mathrm{Ni}$ was nearly half that of SWCNT over the same temperature 


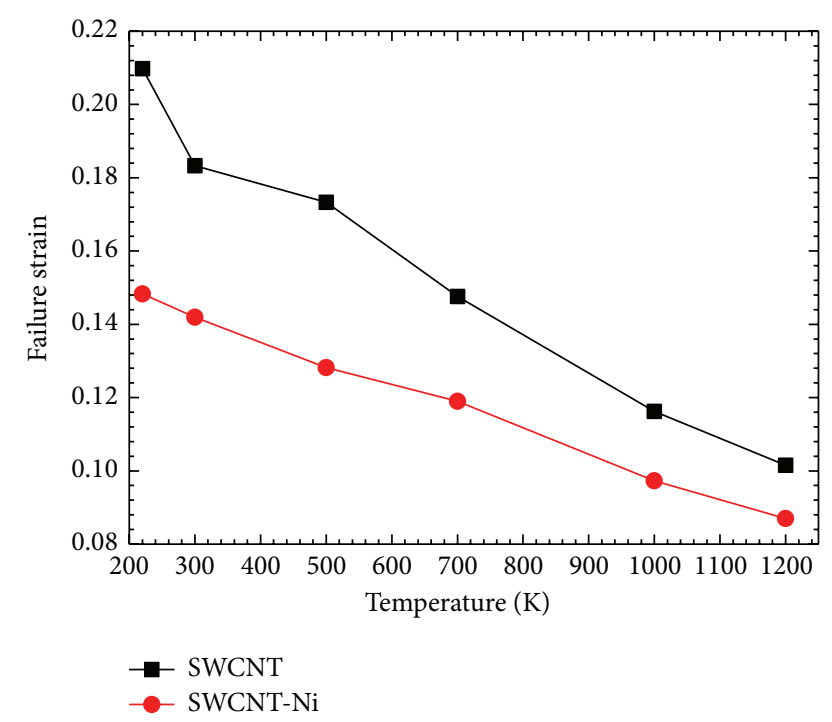

FIGURE 7: Failure strain versus temperature curves of SWCNT and SWCNT-Ni.
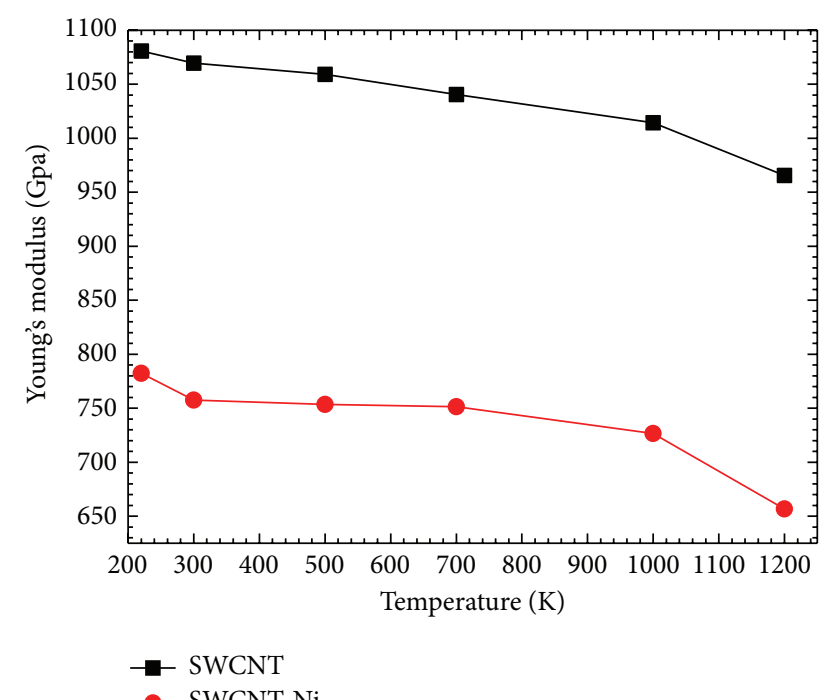

FIGURE 8: Young's modulus versus temperature curves of SWCNT and SWCNT-Ni.

range, which was also found in a previous study [11], and it could be attributed partly to the increase of the crosssectional diameter and the decrease of the failure force of the SWCNT-Ni. Compared with SWCNT, the nickel atoms on the surface of SWCNT-Ni could weaken the strength of SWCNT. When being stretched, the six-member ring of SWCNT-Ni suffered local distortion, the C-C bond length became longer along the $z$-axis (tensile direction), and the $\mathrm{C}-\mathrm{C}$ interaction became equal to the $\mathrm{C}-\mathrm{Ni}$ interaction, which enabled thermal fluctuation to be a key factor for determining the rupture of SWCNT-Ni. In this work, the MD simulation of SWCNT-Ni was different from those using the pristine Brenner potential and the dissimilar attenuation distance [11].
For the failure strains shown in Figure 7, the failure strain of SWCNT was still larger than the experimental results of $10-13 \%$ failure strain [18] and $25 \%$ smaller than those results [20]. Figure 7 clearly shows that the failure strain of SWCNT and SWCNT-Ni would decrease almost linearly with temperature, thus indicating that the effect of temperature is dominant when the temperature increases. During the stretching processes, carbon atoms could prefer to exhibit a $\mathrm{sp}^{2}$ structure and tend to maintain this structure at the Brenner potential [21]. For the stretching of SWCNT-Ni, once a particular $\mathrm{C}-\mathrm{C}$ bond is broken, the nearby nickel atoms would terminate the dangling bonds of the carbon atoms and form bonds with each other [11]. Unlike the failure stress and failure strain shown in Figures 6 and 7, Young's modulus of SWCNT and SWCNT-Ni was not severely sensitive to temperature. In Figure 8, the variation of Young's modulus of SWCNT was approximately $6 \%$ from $220 \mathrm{~K}$ to $1000 \mathrm{~K}$, which was consistent with the results of [22]; however, when the temperature increased to $1200 \mathrm{~K}$, Young's modulus of SWCNT decreased by approximately $10.6 \%$. In Figure 8, all Young's modulus values of SWCNT at temperatures from $220 \mathrm{~K}$ to $1200 \mathrm{~K}$ were larger than the results of [11]. However, compared to those simulations using the Brenner potential function [22] and the modified Morse potential function [15, 23], in this work, Young's modulus of SWCNT was in the range of the computed and experimental values of [18]. After coating nickel onto the surface of SWCNT, Young's modulus of SWCNT-Ni decreased over the temperature range from 220 to $1200 \mathrm{~K}$ by approximately $27.6 \%$. Apparently, the sectional diameter of SWCNT-Ni was larger than that of SWCNT, and the $\mathrm{C}-\mathrm{Ni}$ interactions decreased the $\mathrm{C}-\mathrm{C}$ bond strength, thereby affecting the mechanical behavior of SWCNT-Ni.

When SWCNT or SWCNT-Ni was stretched to some extent, rupturing failure was inevitable. As shown in Figure 9, all the final rupture surfaces at different temperatures were found to be completely brittle, in agreement with the past report [15]. From the stretching rupture surfaces shown in Figure 9, the rupture surfaces of SWCNT-Ni at different temperatures were completely separated, while the rupture surfaces of SWCNT were slightly connected by a single chain of carbon atoms after breaking failure; similar rupture surfaces were also noted in the past [24]. During the stretching simulation for SWCNT and SWCNT-Ni, when breakage of the $\mathrm{C}-\mathrm{C}$ bonds occurred, a significant amount of elastic potential energy was released. As the temperature increased, nickel atoms would obtain enough energy to overcome the energy barrier, thereby breaking the nearby $\mathrm{C}-\mathrm{C}$ bonds, followed by the occurrence of rupture, with the rupture surfaces $45^{\circ}$ from the $z$-direction, as shown Figure 9.

In the MD simulations of stretching SWCNT and SWCNT-Ni at different temperature ranging from 220 to $1200 \mathrm{~K}$, the initial working length, that is, the initial equilibrium total length minus the length of the fixed layer of carbon atoms, could also indicate the effect of temperature on thermal expansion behaviors. The initial working lengths of SWCNT and SWCNT-Ni shown in Figure 10 indicated that the working length of SWCNT-Ni was longer than that of SWCNT at the same temperature, the equilibrium bond 

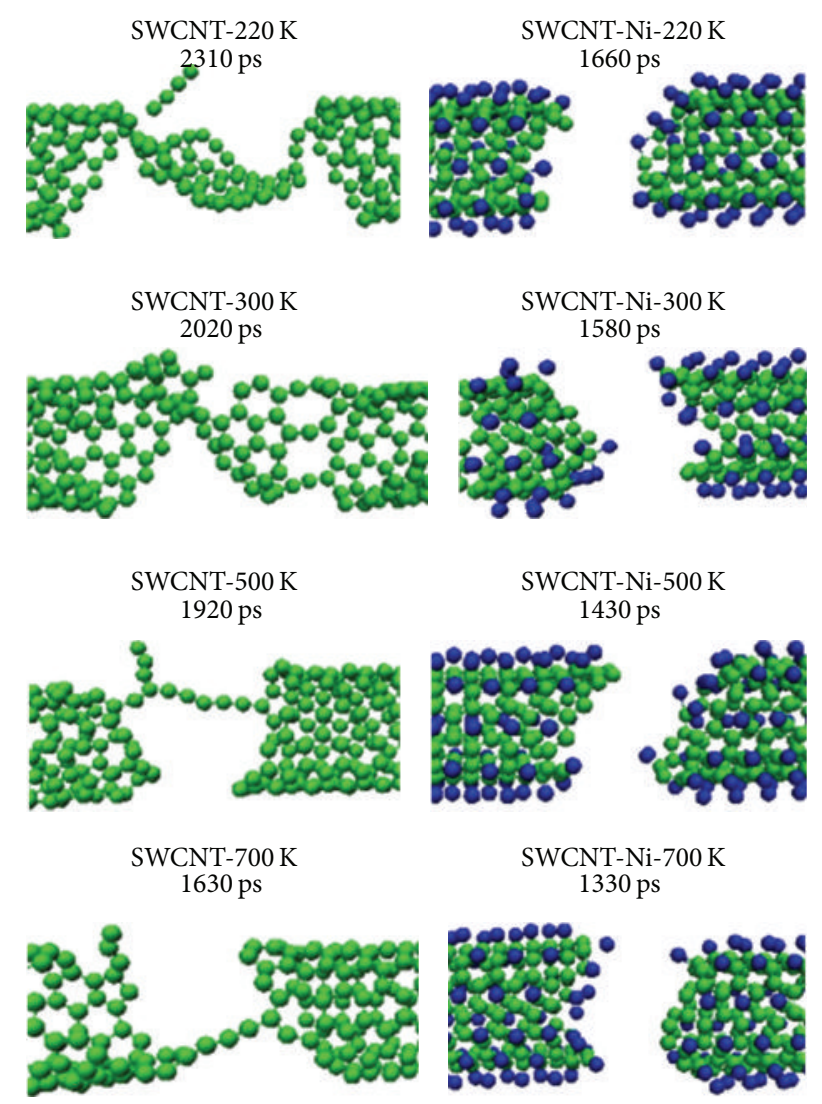

SWCNT-Ni-700 K $1330 \mathrm{ps}$
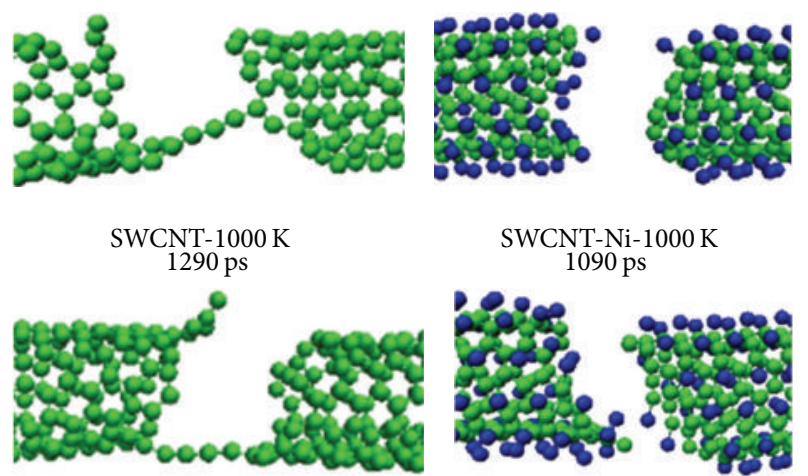

SWCNT-Ni-1000 K $1090 \mathrm{ps}$

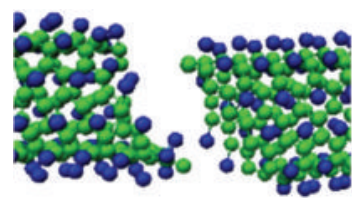

SWCNT-1200 K $1130 \mathrm{ps}$

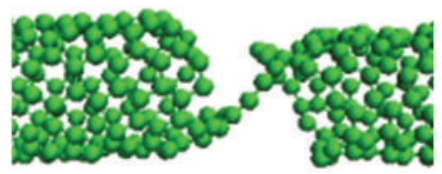

SWCNT-Ni-1200 K $980 \mathrm{ps}$

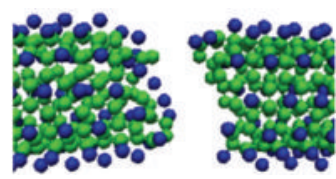

FIGURE 9: Rupture surfaces of SWCNT and SWCNT-Ni at different temperatures.

length of the nickel atoms increased with temperature, and the SWCNT-Ni had a larger thermal expansion coefficient; therefore, SWCNT-Ni was more temperature sensitive than SWCNT. At higher temperature during stretching, the C$\mathrm{C}, \mathrm{C}-\mathrm{Ni}$ and $\mathrm{Ni}-\mathrm{Ni}$ interactions were weakened, leading to the diversification of the mechanical properties, while the nickel atoms on the surface of the SWCNT-Ni played a role in interfering with the $\mathrm{C}-\mathrm{C}$ bonds at a variety of temperatures.

\section{Conclusions}

In this work, using the second-generation Brenner reactive empirical bond-order potential with a modified attenuation

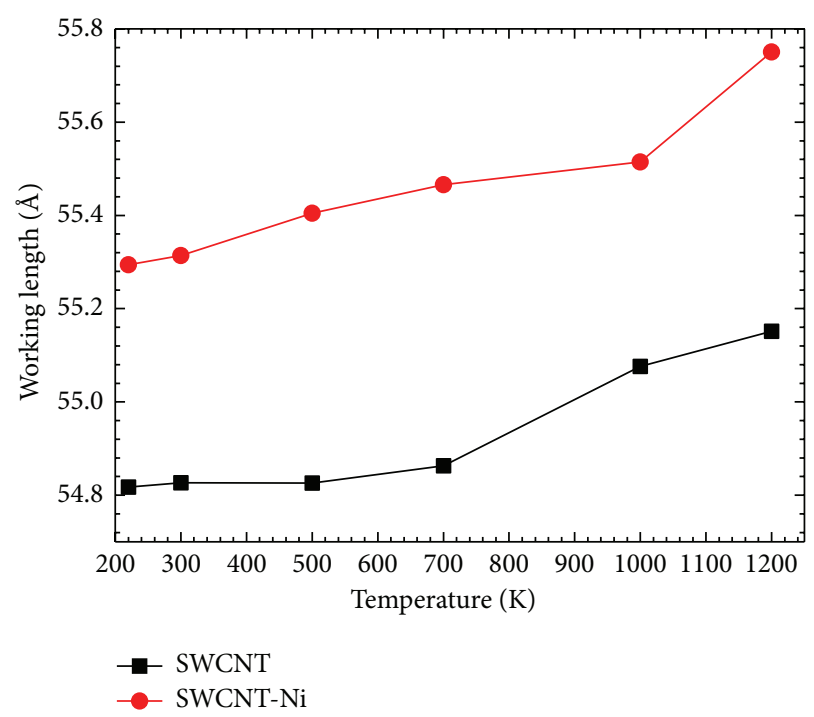

FIGURE 10: Initial working lengths of SWCNT and SWCNT-Ni at different temperatures.

distance of the attenuation function of the Brenner potential, MD simulations of stretching the armchair $(6,6)$ SWCNT and the SWCNT-Ni were conducted at different temperatures of $220 \mathrm{~K}, 300 \mathrm{~K}, 500 \mathrm{~K}, 700 \mathrm{~K}, 1000 \mathrm{~K}$, and $1200 \mathrm{~K}$. The simulation results revealed that the mechanical properties of both SWCNT and SWCNT-Ni were influenced by temperature. In particular, the failure stress and failure strain of SWCNT were obviously reduced at higher temperature, but Young's modulus of SWCNT was not very sensitive to temperature; however, when the temperature was above $1000 \mathrm{~K}$, Young's modulus became greatly reduced. Compared with SWCNT, Young's modulus of SWCNT-Ni was lower at the same temperature. Meanwhile, the failure force and the failure stress of SWCNT and SWCNT-Ni were greatly weakened at higher temperatures. When the surface of SWCNT was coated with nickel atoms, the nickel atoms tended to bind to the carbon atoms. With increasing temperature, nickel atoms on the surface of SWCNT-Ni became more active, and the influence of the nickel interference with the $\mathrm{C}-\mathrm{C}$ bond became more significant, thereby leading to increased temperature sensitivity of the mechanical properties of SWCNTNi.

\section{Conflict of Interests}

The authors declare that there is no conflict of interests regarding the publication of this paper.

\section{Acknowledgments}

This work is supported by 973 project (no. 2015CB057203) and the Fundamental Research Funds for the Central Universities, HUST: 2011TS066, 2013TS023. Technical assistance and suggestions from Dr. Dong Lin of Purdue University are greatly appreciated. 


\section{References}

[1] S. Iijima, "Helical microtubules of graphitic carbon," Nature, vol. 354, no. 6348, pp. 56-58, 1991.

[2] P. Kim, L. Shi, A. Majumdar, and P. L. McEuen, "Thermal transport measurements of individual multiwalled nanotubes," Physical Review Letters, vol. 87, no. 21, 4 pages, 2001.

[3] S. Berber, Y. K. Kwon, and D. Tománek, "Unusually high thermal conductivity of carbon nanotubes," Physical Review Letters, vol. 84, no. 20, pp. 4613-4616, 2000.

[4] J. Xu and T. S. Fisher, "Enhancement of thermal interface materials with carbon nanotube arrays," International Journal of Heat and Mass Transfer, vol. 49, no. 9-10, pp. 1658-1666, 2006.

[5] J. N. Coleman, U. Khan, W. J. Blau, and Y. K. Gun'ko, "Small but strong: a review of the mechanical properties of carbon nanotube-polymer composites," Carbon, vol. 44, no. 9, pp. 1624$1652,2006$.

[6] Y. Zhang, N. W. Franklin, R. J. Chen, and H. Dai, "Metal coating on suspended carbon nanotubes and its implication to metaltube interaction," Chemical Physics Letters, vol. 331, no. 1, pp. 3541, 2000.

[7] H. M. Duong, K. Ishikawa, J. Okawa et al., "Mechanism and optimization of metal deposition onto vertically aligned singlewalled carbon nanotube arrays," Journal of Physical Chemistry C, vol. 113, no. 32, pp. 14230-14235, 2009.

[8] S. Inoue and Y. Matsumura, "Molecular dynamics simulation of physical vapor deposition of metals onto a vertically aligned single-walled carbon nanotube surface," Carbon, vol. 46, no. 15, pp. 2046-2052, 2008.

[9] C. Kim, B. Lim, B. Kim et al., "Strengthening of copper matrix composites by nickel-coated single-walled carbon nanotube reinforcements," Synthetic Metals, vol. 159, no. 5-6, pp. 424-429, 2009.

[10] P. G. Collins, K. Bradley, M. Ishigami, and A. Zettl, "Extreme oxygen sensitivity of electronic properties of carbon nanotubes," Science, vol. 287, no. 5459, pp. 1801-1804, 2000.

[11] S. Inoue and Y. Matsumura, "Influence of metal coating on single-walled carbon nanotube: molecular dynamics approach to determine tensile strength," Chemical Physics Letters, vol. 469, no. 1-3, pp. 125-129, 2009.

[12] Y. Shibuta and S. Maruyama, "Bond-order potential for transition metal carbide cluster for the growth simulation of a singlewalled carbon nanotube," Computational Materials Science, vol. 39, no. 4, pp. 842-848, 2007.

[13] http://lammps.sandia.gov/.

[14] S. J. Stuart, A. B. Tutein, and J. A. Harrison, "A reactive potential for hydrocarbons with intermolecular interactions," Journal of Chemical Physics, vol. 112, no. 14, pp. 6472-6486, 2000.

[15] T. Belytschko, S. P. Xiao, G. C. Schatz, and R. S. Ruoff, "Atomistic simulations of nanotube fracture," Physical Review B: Condensed Matter and Materials Physics, vol. 65, no. 23, 8 pages, 2002.

[16] M. Sammalkorpi, A. Krasheninnikov, A. Kuronen, K. Nordlund, and K. Kaski, "Mechanical properties of carbon nanotubes with vacancies and related defects," Physical Review B, vol. 70, pp. 245416-245423, 2004.

[17] W. J. Chang, "Molecular-dynamics study of mechanical properties of nanoscale copper with vacancies under static and cyclic loading," Microelectronic Engineering, vol. 65, no. 1-2, pp. 239246, 2002.
[18] M.-F. Yu, O. Lourie, M. J. Dyer, K. Moloni, T. F. Kelly, and R. S. Ruoff, "Strength and breaking mechanism of multiwalled carbon nanotubes under tensile load," Science, vol. 287, no. 5453, pp. 637-640, 2000.

[19] S. L. Mielke, D. Troya, S. Zhang et al., "The role of vacancy defects and holes in the fracture of carbon nanotubes," Chemical Physics Letters, vol. 390, no. 4-6, pp. 413-420, 2004.

[20] Y.-R. Jeng, P.-C. Tsai, and T.-H. Fang, "Effects of temperature and vacancy defects on tensile deformation of single-walled carbon nanotubes," Journal of Physics and Chemistry of Solids, vol. 65, no. 11, pp. 1849-1856, 2004.

[21] K. Talukdar and A. K. Mitra, "Comparative MD simulation study on the mechanical properties of a zigzag single-walled carbon nanotube in the presence of Stone-Thrower-Wales defects," Composite Structures, vol. 92, no. 7, pp. 1701-1705, 2010.

[22] N. Yao and V. Lordi, "Young's modulus of single-walled carbon nanotubes," Journal of Applied Physics, vol. 84, no. 4, pp. 19391943, 1998.

[23] J.-P. Salvetat, G. A. D. Briggs, J.-M. Bonard et al., "Elastic and shear moduli of single-walled carbon nanotube ropes," Physical Review Letters, vol. 82, no. 5, pp. 944-947, 1999.

[24] L. G. Zhou and S. Q. Shi, "Molecular dynamic simulations on tensile mechanical properties of single-walled carbon nanotubes with and without hydrogen storage," Computational Materials Science, vol. 23, no. 1-4, pp. 166-174, 2002. 

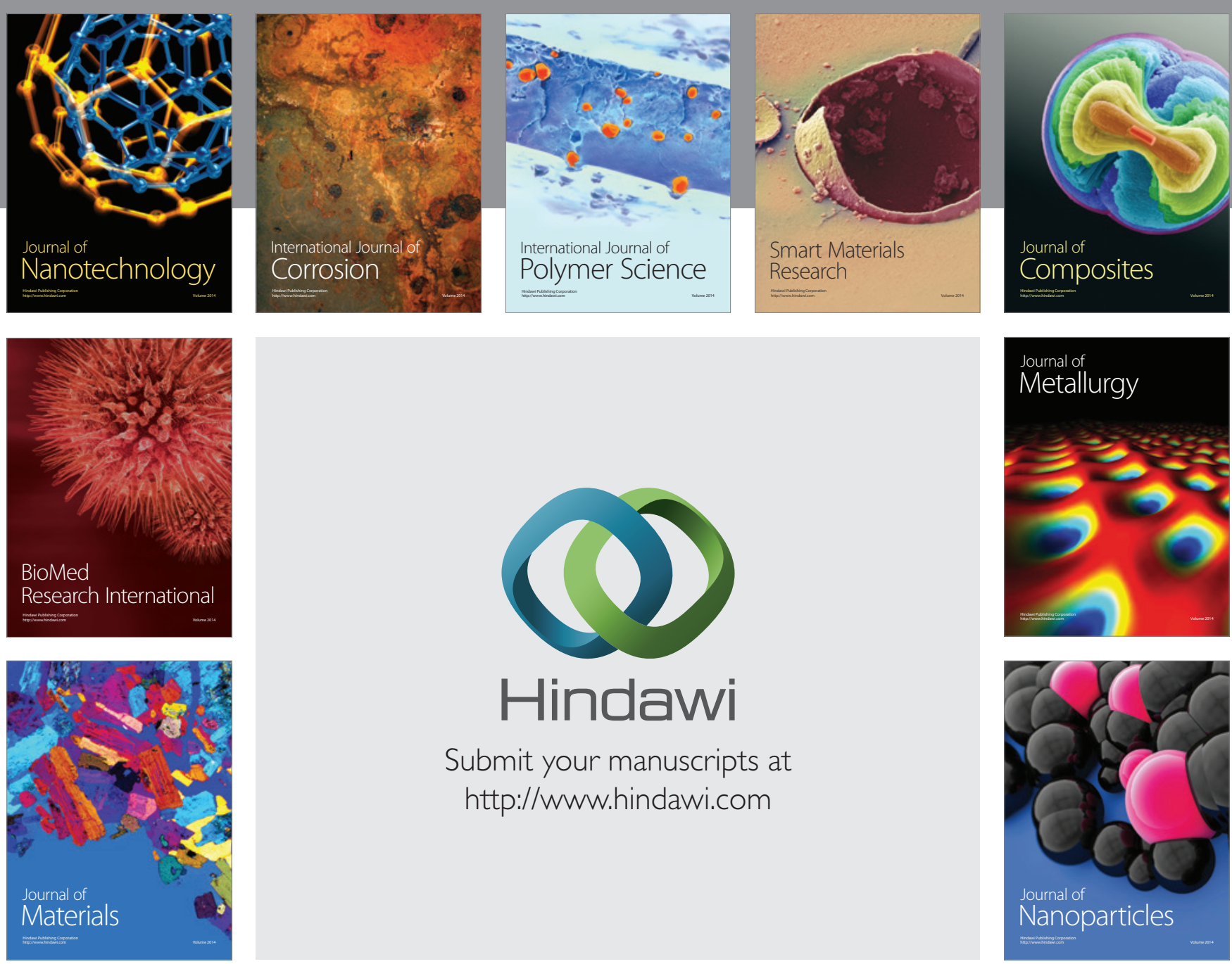

Submit your manuscripts at http://www.hindawi.com
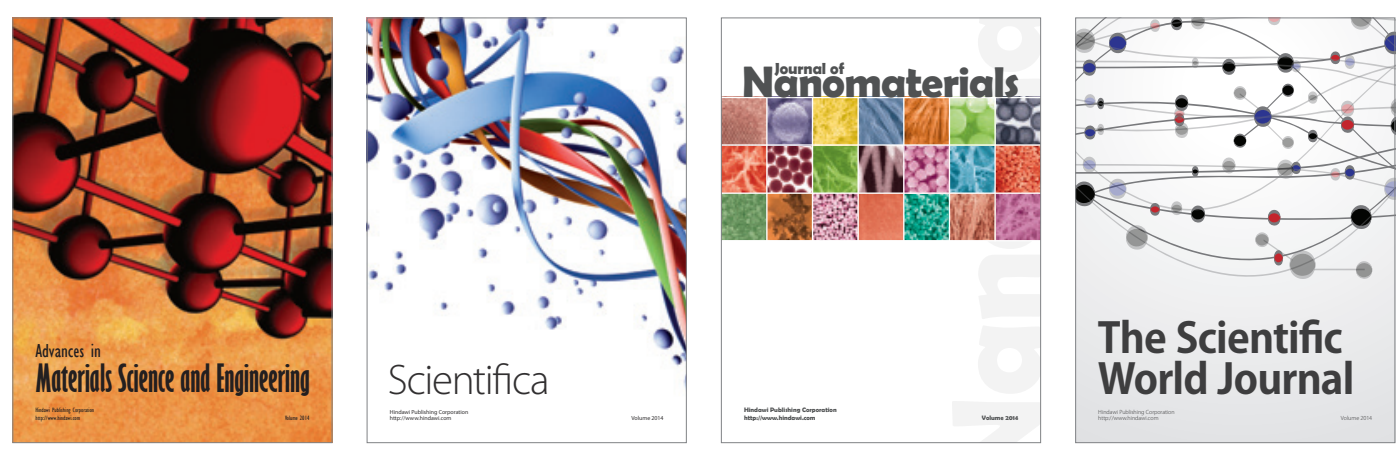

\section{The Scientific World Journal}
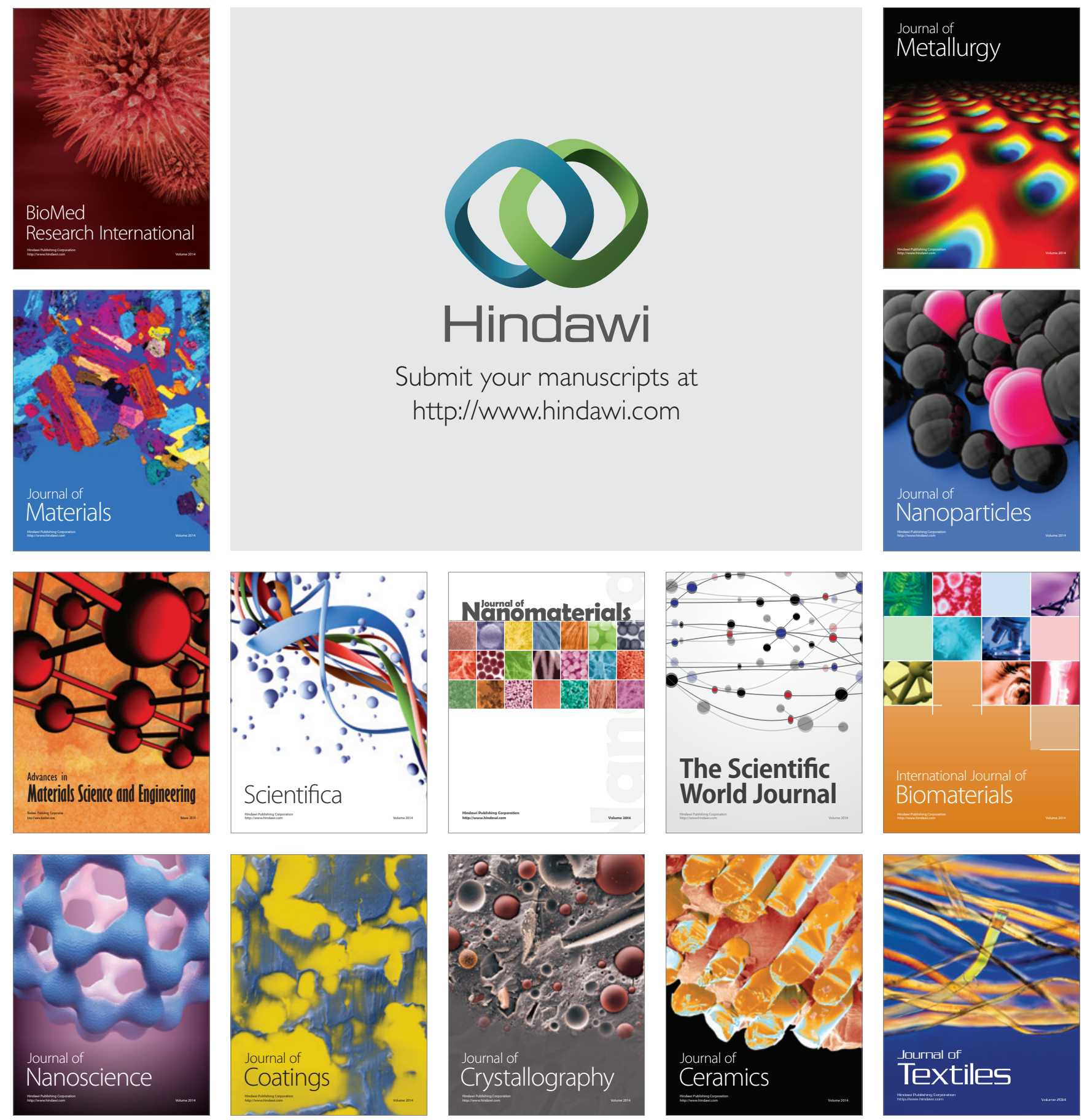